\title{
A note on a theorem of S. Kurepa
}

\author{
By A. K. MOOKHOPADHYAYA
}

(Received June 14, 1962)

(Revised July 23, 1962)

Let $E^{n}$ be the $n$-dimensional Euclidean space, which we interpret also as a vector space in the usual manner. Given a point $c \in E^{n}$ and a positive number $\rho$, we shall denote by $K(c, \rho)$ the closed sphere of centre $c$ and radius $\rho$, i. e. the set of the points $x$ such that $|x-c| \leqq \rho$.

In his illuminating paper [1] , S. Kurepa has recently proved the following result :

THEOREM. Let $P$ be a measurable set in $E^{n}$, and $\alpha_{1}, \alpha_{2}, \cdots, \alpha_{t}$ any finite sequence of real numbers such that

$$
0<\left|\alpha_{k}\right| \leqq 1 \text { for } k=1,2, \cdots, t .
$$

If $x_{0}$ is a point of density for the set $P$, there exist two concentric spheres $K\left(x_{0}, r\right)$ and $K\left(x_{0}, r^{\prime}\right)$ of centre $x_{0}$ such that $r \geqq r^{\prime}$ and that with each point $x$ of the smaller sphere $K\left(x_{0}, r^{\prime}\right)$ we can associate a non-vanishing vector $h(x) \in E^{n}$ so as to fulfil

$$
x+\alpha_{k} h(x) \in K\left(x_{0}, r\right) \cap P \text { for } k=1,2, \cdots, t .
$$

It may be remarked that the conditions $\left|\alpha_{k}\right| \leqq 1$ above are only expediential. The assertion clearly remains valid for any finite sequence of real numbers $\alpha_{1}, \alpha_{2}, \cdots, \alpha_{t}$ other than 0 .

The proof of the theorem, as given by Kurepa, involves some properties of characteristic functions of sets. But the author has found out that the theorem admits of a simpler proof which does not use such a tool. To show this is the object of the present note. By the way, it will turn out in the course of our proof that the radii $r$ and $r^{\prime}$ of the assertion can be so chosen that $r=2 r^{\prime}$.

Notations. (i) The Lebesgue measure of any measurable set $X$ in $E^{n}$ will be written $m(X)$. (ii) For any set $Y \subset E^{n}$ and any real number $\alpha$, we shall denote by $\alpha Y$ the set of the vectors $\alpha y$ where $y$ ranges over $Y$; so that, if $Y$ is in particular measurable, then $m(\alpha Y)=|\alpha|^{n} m(Y)$. (iii) If $U$ and $V$ are two sets in $E$, then by $U \backslash V$ we shall understand the set of the points of $U$ which are not in $V$, and by $U-V$ the set of all the vectors $u-v$ where $u \in U$ and $v \in V$. 
Proof of The Theorem. To shorten our notations, we put $\beta_{k}=1 / \alpha_{k}$ for every $k=1,2, \cdots, t$ and further $\delta=2^{n}\left(\left|\beta_{1}\right|^{n}+\cdots+\left|\beta_{t}\right|^{n}\right)$. By hypothesis there exists a sphere $K_{0}=K\left(x_{0}, r\right)$ with centre $x_{0}$ and radius $r$, such that $\delta m\left(K_{0} \backslash Q\right)<m\left(K_{0}\right)$, where we write $Q=K_{0} \cap P$.

Let $S(x)=\left[\beta_{1}(Q-x)\right] \cap \cdots \cap\left[\beta_{t}(Q-x)\right]$ for each $x \in K\left(x_{0}, s\right)$, where $s=r / 2$. We shall show that this set $S(x)$ is always of strictly positive measure. Suppose, if possible, that $m[S(y)]=0$ for some $y \in K\left(x_{0}, s\right)$. It is then obvious that

$$
m[K(0, s)]=m[K(0, s) \backslash S(y)] \leqq \Sigma m\left[K(0, s) \backslash \beta_{k}(Q-y)\right],
$$

where and subsequently the summation extends over $k=1,2, \cdots, t$. But here $K(0, s) \subset K\left(0,\left|\beta_{k}\right| s\right)=\beta_{k} K(0, s)$ for every $k$, since $\left|\beta_{k}\right| \geqq 1$. Consequently we find that

$$
\begin{aligned}
m[K(0, s)] & \leqq \sum m\left[\beta_{k} K(0, s) \backslash \beta_{k}(Q-y)\right] \\
& =\Sigma\left|\beta_{k}\right|^{n} \cdot m[K(0, s) \backslash(Q-y)] \\
& =2^{-n} \delta \cdot m[K(y, s) \backslash Q] \leqq 2^{-n} \delta \cdot m\left(K_{0} \backslash Q\right),
\end{aligned}
$$

the last inequality being a consequence of the inclusion $K(y, s) \subset K_{0}$. In view of our choice of the sphere $K_{0}$, this leads at once to

$$
2^{n} \cdot m[K(0, s)]<m\left(K_{0}\right)=m[K(0, r)],
$$

which, however, contradicts the relation $r=2 s$.

Thus, for every $x \in K\left(x_{0}, s\right)$, we must have $m[S(x)]>0$ and so the set $S(x)$ certainly contains a vector $h(x) \neq 0$. This completes the proof.

Finally the author is greatly thankful to Dr. B.K. Lahiri for his helpful suggestions and discussions and to the referee for the valuable help he has rendered in improving the paper.

\section{Charuchandra College Calcutta, India}

\section{Reference}

[1] S. Kurepa, A property of a set of positive measure and its application, J. Math. Soc. Japan, 13 (1961), 13-19. 